\title{
Condilectomia alta associada à cirurgia ortognática para tratamento de hiperplasia condilar ativa: relato de caso
}

\author{
High condylectomy associated with orthognathic surgery for the treatment \\ of active condylar hyperplasia: case report \\ Condilectomía alta asociada a la cirugía ortognática para tratamiento \\ de hiperplasia condilar activa: relato de caso \\ Leandro da Cunha DIAS ${ }^{1}$ \\ Caleb Rogério Caetano FERREIRA ${ }^{2}$ \\ ${ }^{1}$ Residente em Cirurgia e Traumatologia Bucomaxilofaciais, Associação Nacional de Estudos Odontológicos, \\ ANEO, 72010-010 Brasília - DF, Brasil \\ ${ }^{2}$ Preceptor da Residência em Cirurgia e Traumatologia Bucomaxilofaciais, Associação Nacional de Estudos Odontológicos, \\ ANEO, 72010-010 Brasília - DF, Brasil
}

\begin{abstract}
Resumo
A hiperplasia condilar é uma doença caracterizada pelo crescimento do côndilo mandibular de forma patológica e progressiva, acarretando seu aumento de tamanho e volume, e cuja etiologia ainda é desconhecida. Quando a hiperplasia encontra-se em atividade, abordagem cirúrgica pode ser necessária para remoção do centro de crescimento condilar ativo. O objetivo deste trabalho é relatar o caso de uma hiperplasia condilar unilateral ativa em uma paciente jovem do sexo feminino, que foi tratada por condilectomia alta, associada à cirurgia ortognática maxilo-mandibular, para correção de deformidade dentofacial desenvolvida. A paciente encontra-se com um ano de acompanhamento pós-operatório, sem recidiva da hiperplasia e sem sintomatologia articular, com a oclusão estável e resolução da assimetria facial. A condilectomia alta em combinação à cirurgia ortognática, como forma de tratamento da hiperplasia condilar unilateral ativa, mostrou ser eficaz, estável e previsível.

Descritores: Articulação Temporomandibular; Côndilo Mandibular; Disco da Articulação Temporomandibular; Artroplastia; Procedimentos Cirúrgicos Ortognáticos.
\end{abstract}

\begin{abstract}
Condylar hyperplasia is a disease characterized by the growth of the mandibular condyle in a pathological and progressive way, causing its increase of size and volume, and whose etiology still is unknown. When hyperplasia is active, surgical approach may be necessary for removal of active condylar growth center. The objective of this study is to report the case of an active unilateral condylar hyperplasia in a young female patient, who was treated by high condilectomy associated with maxillo-mandibular orthognathic surgery for correction of developed dentofacial deformity. The patient has one year of postoperative follow-up, with no recurrence of hyperplasia and no joint symptomatology, with stable occlusion and facial asymmetry's resolution. High condilectomy in combination with orthognathic surgery, as a form of treatment of active unilateral condylar hyperplasia, was shown effective, stable and predictable.

Descriptors: Temporomandibular Joint; Mandibular Condyle; Temporomandibular Joint Disc; Arthroplasty; Orthognathic Surgical Procedures.
\end{abstract}

\section{Resumen}

La hiperplasia condilar es una enfermedad caracterizada por el crecimiento del cóndilo mandibular de forma patológica y progresiva, acarreando su aumento de tamaño y volumen, y cuya etiología todavía es desconocida. Cuando la hiperplasia se encuentra en actividad, el enfoque quirúrgico puede ser necesario para la remoción del centro de crecimiento condilar activo. El objetivo de este trabajo es relatar el caso de una hiperplasia condilar unilateral activa en una paciente joven del sexo femenino, que fue tratada por una condilectomía alta, asociada a la cirugía ortognática maxilo-mandibular, para corrección de deformidad dentofacial desarrollada. La paciente se encuentra con un año de seguimiento postoperatorio, sin recidiva de la hiperplasia y sin sintomatología articular, con la oclusión estable y resolución de la asimetría facial. La condilectomía alta en combinación con la cirugía ortognática, como forma de tratamiento de la hiperplasia condilar unilateral activa, mostró ser eficaz, estable y previsible.

Descriptores: Articulación Temporomandibular; Cóndilo Mandibular; Disco de la Articulación Temporomandibular; Artroplastia; Procedimientos Quirúrgicos Ortognáticos.

\section{INTRODUÇÃO}

O côndilo é considerado o centro de crescimento primário da mandíbula, e pode ser acometido por anormalidades adquiridas ao longo do desenvolvimento do indivíduo ${ }^{1}$. A hiperplasia condilar (HC) é uma dessas anormalidades, sendo considerada uma desordem não neoplásica de origem desconhecida, na qual há crescimento patológico progressivo do côndilo mandibular, ocasionando aumento do seu tamanho e volume $\mathrm{e}^{2,3}$.

Esta condição é, supostamente, secundária a um centro de crescimento hipermetabólico anormal no côndilo afetado ${ }^{1}$. Sua etiologia ainda é incerta, embora infecções, fatores genéticos, traumatismos, distúrbios hormonais e problemas microcirculatórios locais possam estar associados ${ }^{2,4}$.

Sua manifestação clínica pode incluir assimetria facial (laterognatismo contralateral), prognatismo, mordida cruzada e aberta posterior, dor e, ocasionalmente, um crescimento maxilar compensatório com inclinação do plano oclusal $(\text { cant })^{3}$. Radiograficamente, pode-se notar um alongamento do colo e do côndilo, ou de todo o ramo mandibular.

A cintilografia óssea avalia o nível de metabolismo ósseo, indicando se a HC está em atividade ou não. Uma diferença de atividade celular de mais de $10 \%$ entre os côndilos direito e esquerdo, captada na cintilografia óssea, é fortemente sugestiva de hiperplasia condilar, ${ }^{3,5}$.

Pacientes com atividade condilar hiperplásica podem ser submetidos, como forma de tratamento, à condilectomia alta, com ressecção do centro de crescimento cartilaginoso, associada ou não à cirurgia ortognática. $\mathrm{O}$ objetivo deste trabalho é relatar um caso clínico de hiperplasia condilar unilateral ativa (HCUA) tratada por condilectomia alta associada à cirurgia ortognática bimaxilar.

\section{CASO CLÍNICO}

Paciente do sexo feminino, 26 anos de idade, realizando tratamento ortodôntico, comparece à consulta com história de assimetria facial, desvio de linha média mandibular, sorriso gengival e estalos em articulação temporomandibular (ATM). Ao exame físico, notou-se discreto prognatismo mandibular com laterognatismo à esquerda, assimetria do plano oclusal com inclinação do eixo roll (cant), mordida cruzada posterior do lado esquerdo, estalidos e artralgias bilaterais em ATM (Figura 1). 


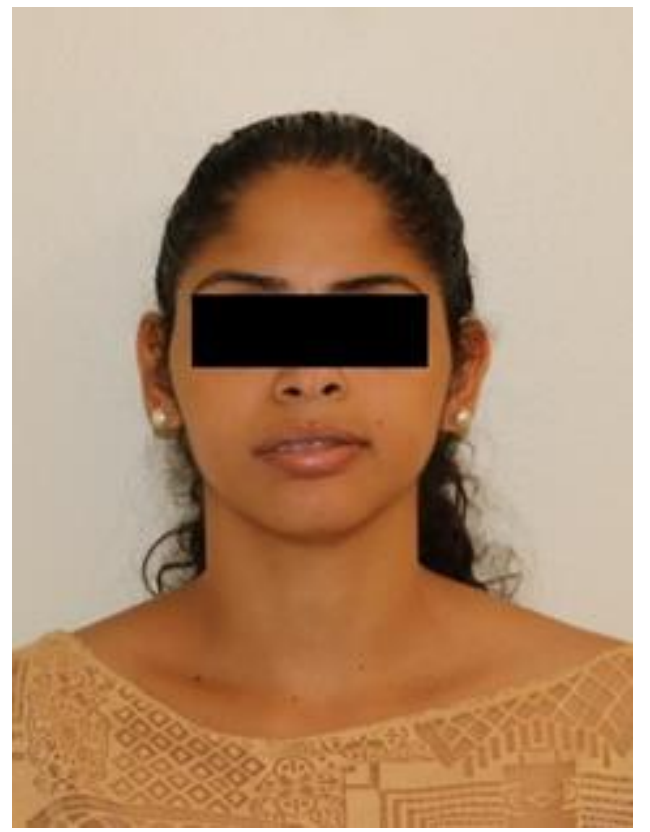

Figura 1: Aspecto pré-operatório. Notar inclinação do plano oclusal (cant) à direita, com desnivelamento das comissuras labiais.

No exame radiográfico panorâmico, pôde-se perceber aumento de tamanho do côndilo mandibular direito. No exame de ressonância magnética, notou-se aumento de volume do côndilo direito com adelgaçamento do disco articular, e deslocamento anterior do disco articular com redução em ambos os lados. Suspeitou-se, então, de hiperplasia condilar unilateral, sendo solicitada à paciente uma cintilografia óssea, a qual foi positiva para crescimento condilar ativo à direita.

Com base no exame clínico e nos exames complementares, chegou-se ao diagnóstico de HCUA, deslocamento anterior bilateral de discos articulares e deformidade dentofacial. $\mathrm{O}$ tratamento preconizado foi a condilectomia alta no lado direito, reposicionamento e plicatura bilateral dos discos articulares e, por fim, cirurgia ortognática maxilo-mandibular com reposicionamento mentual, realizada através de planejamento virtual no software Dolphin 3D Surgery®.

$\mathrm{O}$ ato cirúrgico iniciou pelas articulações temporomandibulares, seguido pela mandíbula, mento e maxila. O acesso cirúrgico de escolha às ATM's foi o endaural, sendo realizada a condilectomia alta, no lado direito, através da ressecção de $5 \mathrm{~mm}$ de todo o aspecto mais superior do côndilo, desde o polo lateral ao medial, o qual corresponde à zona de crescimento condilar ativo. Em seguida, o disco articular foi estabilizado em sua posição anatômica através de plicatura com fio de poliéster não absorvível (Ethibond ${ }^{\mathrm{TM}}$ ). Esse mesmo procedimento de plicatura discal foi realizado no lado esquerdo para tratar o deslocamento anterior do disco articular (Figuras 2, 3 e 4).

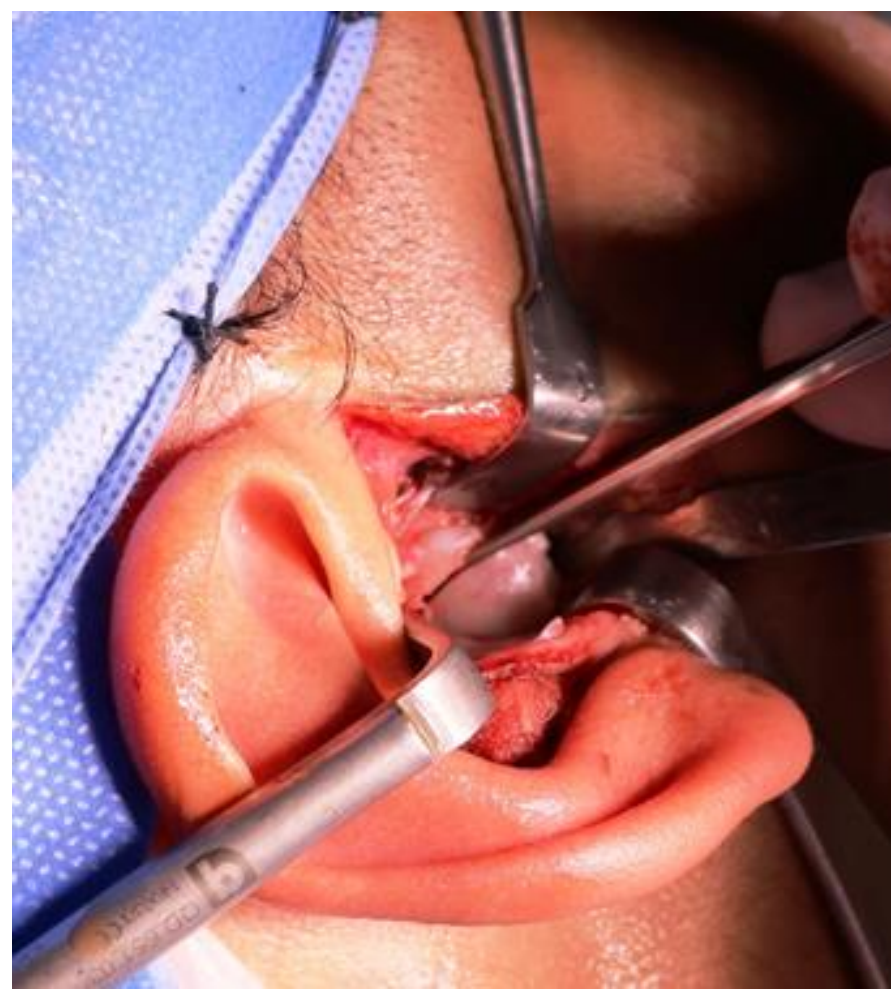

Figura 2: Condilectomia alta.

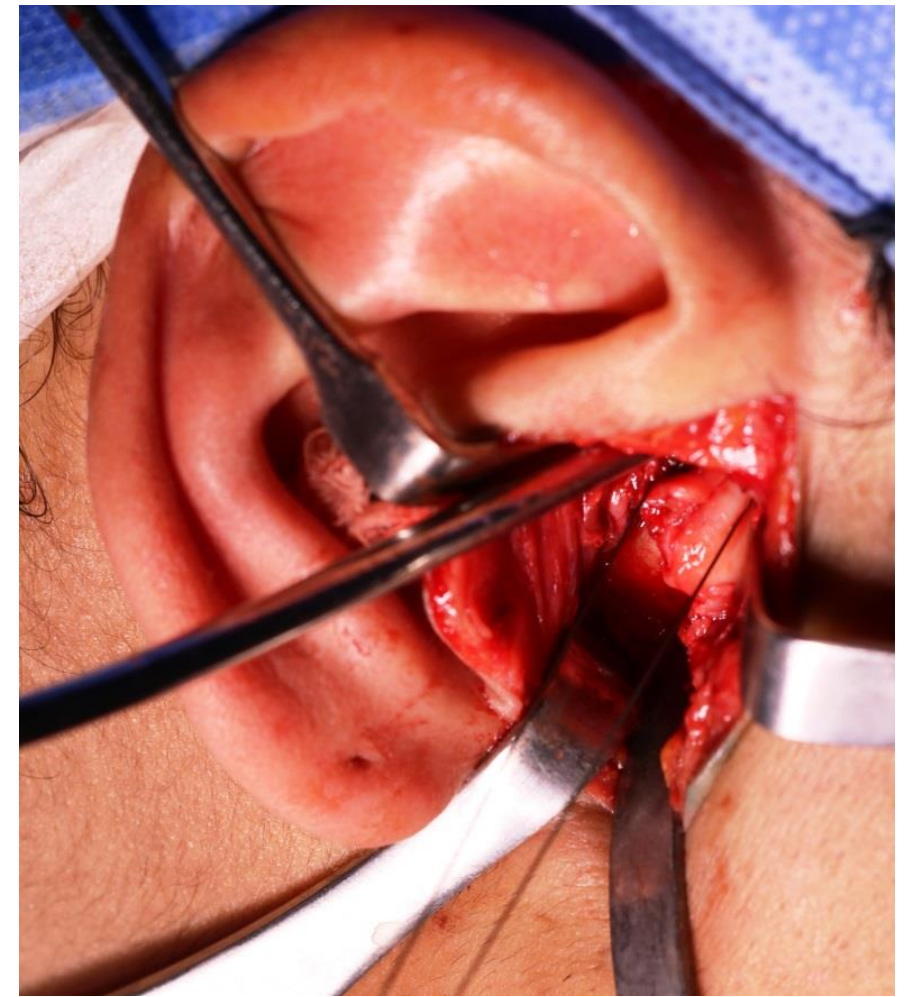

Figura 3: Plicatura do disco articular sendo realizada.

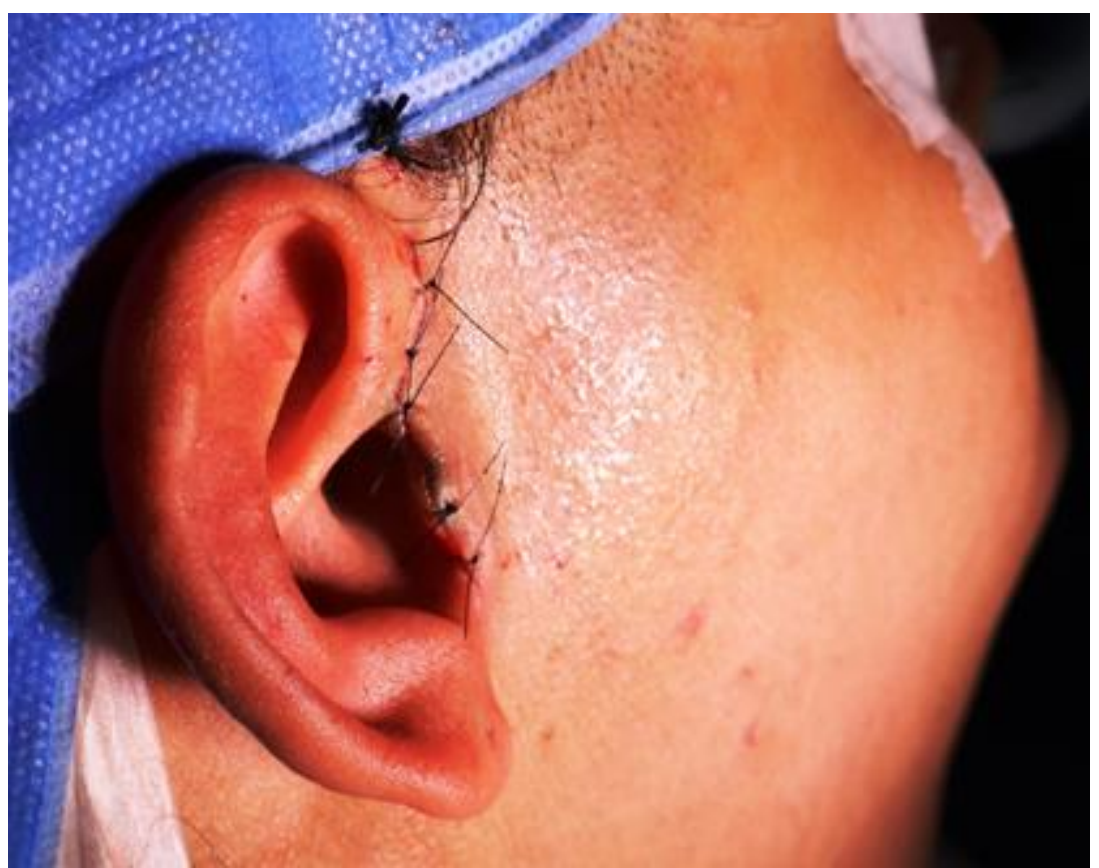

Figura 4: Sutura e aspecto final do acesso endaural.

Após a cirurgia da ATM, partiu-se para a correção da deformidade dentofacial através da cirurgia ortognática, iniciando-se com osteotomia sagital dos ramos mandibulares bilateralmente, seguido por osteotomia horizontal basilar do mento e, por último, osteotomia Le Fort I com segmentação de maxila (Figura 5).

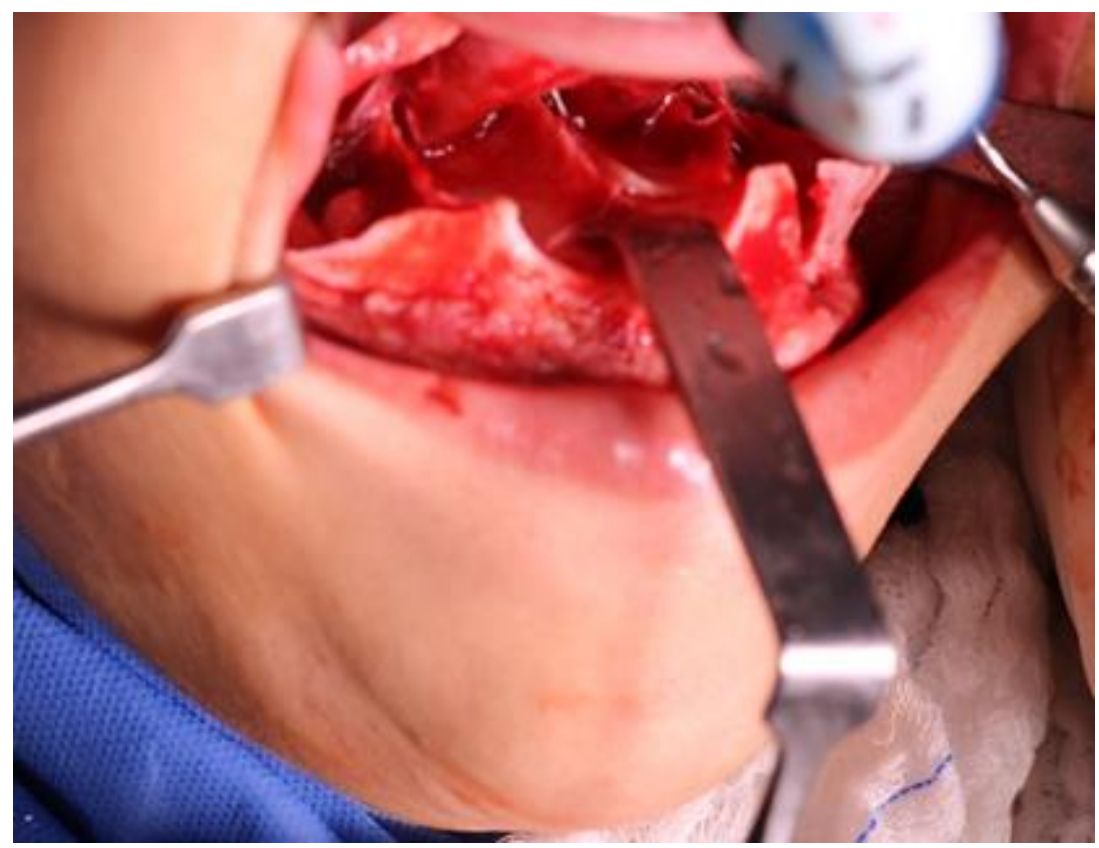

Figura 5: Osteotomia Le Fort I com segmentação de maxila.

Após um ano de acompanhamento pós-operatório a paciente não apresentou recidiva da hiperplasia, sons ou dores articulares, queixas ou complicações, apresentando oclusão estável e resolução da assimetria facial (Figura 6). 


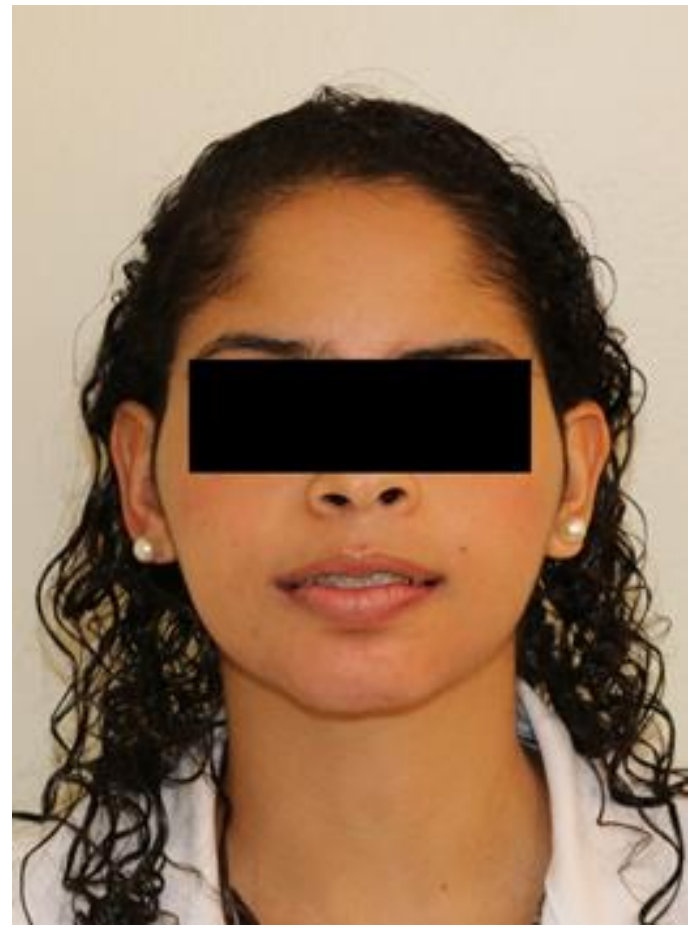

Figura 6: Foto pós-operatória de um ano de acompanhamento. Notar comissuras labiais niveladas devido à correção do plano oclusal.

\section{DISCUSSÃO}

A hiperplasia condilar ocorre mais frequentemente em pacientes do sexo feminino do que masculino ${ }^{1,2,4}$, e teorias sugerem estar relacionada a influências estrogênicas ${ }^{2}$.

Segundo Mouallem ${ }^{2}$ e Wolford et al. ${ }^{6}$, a hiperplasia condilar ativa é uma doença que se estabelece precocemente, entre a infância e a segunda década de vida, o que é corroborado no caso aqui apresentado. Ademais, Mouallem ${ }^{2}$ ainda cita, em revisão feita de 73 casos de hiperplasia condilar unilateral, que a idade média de diagnóstico/intervenção é de $23 \pm 10$ anos, e que a maioria $(72,7 \%)$ apresentou resultado positivo para crescimento condilar ativo quando submetida ao exame de cintilografia óssea, sendo maior a predileção pelo côndilo direito. Trabalhos de Wolford et al. ${ }^{7}$ em 2002 e de Wolford et al. ${ }^{6}$ em 2009 ainda destacam que a cintilografia óssea pode ser mais efetiva em casos unilaterais, especialmente se for realizada após o surto de crescimento ósseo normal, quando se espera que o crescimento condilar tenha cessado. Todos esses dados apresentados por esses autores condizem com o relato aqui apresentado.

O tratamento da hiperplasia condilar depende da apresentação clínica da doença e se ela está ativa ou não. Os objetivos do tratamento são eliminar o processo patológico e fornecer um ótimo benefício estético e funcional ao paciente. Para a forma ativa dessa condição, é indicada a remoção cirúrgica do centro de crescimento condilar (condilectomia alta) combinado à cirurgia ortognática, se necessário ${ }^{1}$.

Para Chouinard et al. ${ }^{1}$, Mouallem et al. ${ }^{2}$, Ghawsi et al. ${ }^{4}$ e Wolford et al. ${ }^{6}$, na condilectomia alta, uma ressecção média de $5 \mathrm{~mm}$ da porção superior da cabeça condilar, incluindo os polos lateral e medial, é suficiente para completa remoção do centro de crescimento condilar, a fim de se obter um resultado favorável. O caso clínico relatado no presente trabalho está em conformidade com a literatura, visto a quantidade de osso que foi excisado e a estabilidade do caso apresentado, com ausência de recidiva da doença durante o período acompanhado.

Wolford et al. ${ }^{7}$ também apontam que não há influência, em longo prazo, da condilectomia na função articular, desde que o disco articular seja preservado e reposicionado durante a cirurgia, tal qual foi realizado neste trabalho e pôde ser confirmado pela ausência de sintomatologia articular.

Vários autores ${ }^{3,6,7}$ sugerem associação de técnicas cirúrgicas ortognáticas à condilectomia alta para tratar HCUA. Neste caso, optou-se por uma cirurgia bimaxilar para otimizar a função e a estética da paciente. Além disso, a escolha pela osteotomia sagital dos ramos mandibulares se deu porque ela proporciona melhor controle posicional dos côndilos quando comparado às outras técnicas existentes (osteotomia em "L" invertido, osteotomia vertical do ramo) e também por manter máxima adesão de tecidos moles e vascularização do segmento proximal.

\section{CONSIDERAÇÕES FINAIS}

Existe uma relação de causa-efeito entre a hiperplasia condilar ativa unilateral e a deformidade dentofacial, sendo a cirurgia articular aliada à cirurgia ortognática um importante método cirúrgico de tratamento dessa condição.

A hiperplasia condilar ativa unilateral tratada com condilectomia alta, reposicionamento do disco articular e cirurgia ortognática bimaxilar em um mesmo tempo cirúrgico apresentou um resultado estável, previsível e favorável, sendo que as técnicas ortognáticas associadas melhoraram as características estéticas e oclusais.

\section{REFERÊNCIAS}

1. Chouinard AF, Kaban LB, Peacock ZS. Acquired abnormalities of the temporomandibular joint. Oral Maxillofacial Surg Clin North Am. 2018;30(1):83-96.

2. Mouallem G, Vernex-Boukerma Z, Longis J, Perrin JP, Delaire J, Mercier JM et al. Efficacy of proportional condylectomy in a treatment protocol for unilateral condylar hyperplasia: A review of 73 cases. J Craniomaxillofac Surg. 2017;45(7):1083-93.

3. Rodrigues DB, Castro V. Condylar hyperplasia of the temporomandibular joint: types, treatment, and surgical implications. Oral Maxillofacial Surg Clin North Am. 2015;27(1):155-67.

4. Ghawsi S, Aagaard E, Thygesen TH. High condylectomy for the treatment of mandibular condylar hyperplasia: a systematic review of the literature. Int $\mathrm{J}$ Oral Maxillofac Surg. 2016;45(1):60-71.

5. Saridin CP, Raijmakers PG, Tuinzing DB, Becking AG. Bone scintigraphy as a diagnostic method in unilateral hyperactivity of the mandibular condyles: a review and meta-analysis of the literature. Int J Oral Maxillofac Surg 2011;40(1):11-7.

6. Wolford LM, Morales-Ryan CA, Garcia-Morales P, Perez D. Surgical management of mandibular condylar hyperplasia type 1. Proc (Bayl Univ Med Cent) 2009;22(4):321-9.

7. Wolford LM, Mehra P, Reiche-Fischel O, MoralesRyan CA, García-Morales P. Efficacy of High Condylectomy for Management of Condylar Hyperplasia. Am J Orthod Dentofacial Orthop. 2002;121(2):136-50.

\section{CONFLITO DE INTERESSES}

Os autores declaram não haver conflitos de interesse.

\section{AUTOR PARA CORRESPONDÊNCIA}

\section{Leandro da Cunha Dias}

leandrocdias@yahoo.com.br

Submetido em 04/03/2018 Aceito em 09/04/2018 